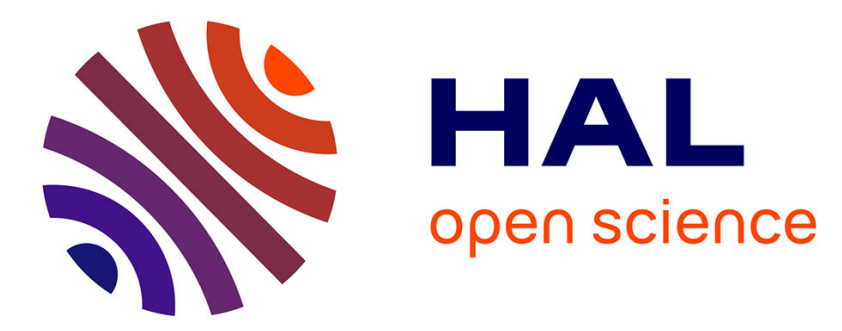

\title{
Comparaison de solveurs numériques pour simuler la turbulence compressible
}

\author{
Emmanuelle Declercq, Alain Forestier, Jean-Marc Hérard
}

\section{To cite this version:}

Emmanuelle Declercq, Alain Forestier, Jean-Marc Hérard. Comparaison de solveurs numériques pour simuler la turbulence compressible. Comptes Rendus de l'Académie des Sciences - Series I - Mathematics, 2000, 331, pp.1011-1016. 10.1016/S0764-4442(00)01745-6 . hal-01580050

\section{HAL Id: hal-01580050 \\ https://hal.science/hal-01580050}

Submitted on 23 Dec 2019

HAL is a multi-disciplinary open access archive for the deposit and dissemination of scientific research documents, whether they are published or not. The documents may come from teaching and research institutions in France or abroad, or from public or private research centers.
L'archive ouverte pluridisciplinaire HAL, est destinée au dépôt et à la diffusion de documents scientifiques de niveau recherche, publiés ou non, émanant des établissements d'enseignement et de recherche français ou étrangers, des laboratoires publics ou privés. 


\title{
Comparaison de solveurs numériques pour simuler la turbulence compressible
}

\author{
Emmanuelle DECLERCQ, Alain FORESTIER et Jean-Marc HÉRARD
}

E. D. : C.E.M.I.F., 40 rue du Pelvoux, Courcouronnes, 91020 Evry Cedex

A. F. : C.E.A. Saclay, DRN/DMT/SEMT, 91191 Gif-Sur-Yvette Cedex

J.-M. H. : E.D.F. Division Recherche et Développement,

Département M.F.T.T., 6 quai Watier, 78400 Chatou

C.M.I., 39 rue Joliot Curie, Université de Provence, 13453 Marseille Cedex 13

Résumé. Nous nous intéressons à la résolution numérique du système hyperbolique décrivant un écoulement turbulent compressible de gaz en situation isentropique. Nous construisons le solveur de Riemann exact associé, nécessaire au schéma de Godunov, que nous comparons au schéma Vfroe et au schéma de Rusanov.

Comparison of numerical solvers for turbulent compressible flow.

Abstract. We focus on the computation of the hyperbolic system describing a turbulent flow for isentropic gas. An exact Riemann solver is computed, we construct the solution of the Riemann problem and thus we derive a Godunov scheme. We provide some numerical simulations to exhibit a comparison between Godunov scheme and two approximate solvers: Vfroe and Rusanov scheme.

\section{Abridged English Version}

In this Note, we focus on the non conservative hyperbolic system arising from the $\mathrm{K}$ turbulent compressible model applied to multicomponent flow and polytropic gases. Variables $\rho, Y, U$ and $K$ denote respectively the mean density of the mixture, the mass fraction of one component in the mixture, the velocity and the kinetic turbulent energy of the mixture $K=\frac{1}{2} \overline{\rho U^{\prime} U^{\prime}}$. Governing equations of the non conservative system are given by (2). We get the entropy-entropy flux pair $\left(\mathcal{E}, f_{\mathcal{E}}\right)=\left(\frac{\rho U^{2}}{2}+K+\frac{P}{\gamma-1}, \mathcal{E} U+\frac{2}{3} K U+\frac{P U}{\gamma-1}\right)$ and by the entropy inequality $\sigma[\mathcal{E}] \geq\left[f_{\mathcal{E}}\right]$ on schock curves of $\sigma$ discontinuity celerity, the unique solution is exhibited. The exact solution of the Riemann Problem enables to implement the Godunov scheme. We refer to Coquel and Berthon [1], [2] for a somewhat different approach to deal with non conservative equations arising in systems with two distinct entropies. An other aim is the comparison between Godunov scheme [10] and two linearized methods. The first one, Vfroe-ncv (see (13)-(17) and [3]), is very close to Godunov method, and thus gives very accurate results. It divides the CPU cost by a factor ten. However, this latter method has some drawbacks, since both pressure and turbulent kinetic energy positivity can not be ensured in all cases. This has been observed, for instance, in some strong rarefaction waves oc- 
curring behind some bluff bodies. The Rusanov scheme (see (19)-(21) and [12]) ensures positivity of the variables $\rho, Y, K$, but this scheme is so diffusive that it provides poorly accurate results. We conclude that in some extreme cases, the cost of an exact solver is justified by the robustness requirement. Anyway, we advocate to use successively two schemes: the exact one for initialization and to deal with the boundary conditions, and a linearized one to go on with a good compromise between accuracy and computational cost.

\section{Introduction}

On considère un écoulement isentropique turbulent de deux composants gazeux. Dans cette étude on s'intéresse particulièrement au couplage pression-turbulence. La turbulence étant supposée isotrope, le tenseur de Reynolds est décrit par l'énergie cinétique turbulente du mélange $K$. La partie convective du système étant dominante, on ne traitera que la fermeture au premier ordre du modèle (cf. [11] pour un modèle plus complet).

\section{Solveur de Riemann exact et schéma de Godunov}

On s'intéresse à la résolution du système portant sur les variables moyennées par le processus de Favre: $\rho$ est la densité du mélange, $U$ la vitesse du mélange, $Y$ la fraction massique d'un des deux composants et $K$ la turbulence sommée des deux fluides. La pression $P$ est connue par la loi d'état $P=P(\rho Y)$. On note $C=(\rho, \rho Y, \rho U)^{t}, W(x, t)=(C, K)^{t}$, avec $x \in \Omega\left(\subset \mathbb{R}^{2}\right), t \geq 0$.

$$
\begin{array}{r}
C=\left(\begin{array}{l}
\rho \\
\rho Y \\
\rho U
\end{array}\right) \quad F(C, K)=\left(\begin{array}{l}
\rho U \\
\rho Y U \\
\rho U \otimes U+\left(\frac{2}{3} K+P\right) I
\end{array}\right) \\
(\mathcal{S})\left\{\begin{array}{l}
\partial_{t} C+\nabla F(C, K)=0 \\
\partial_{t} K+\nabla(K U)+\frac{2}{3} K \nabla U=0
\end{array}\right.
\end{array}
$$

On se ramène au système $\left(\mathcal{S}_{n}\right)$ monodimensionnel, projection de $(2)$ suivant la normale $\vec{n}$ à une interface d'un volume de contrôle. Les composantes normale et tangentielle de $U$ sont désignées par $u_{n}$ et $u_{t}$. Soit $M$ la matrice de projection dans le repère normal, $M W=W_{n}=\left(\rho, \rho Y, \rho u_{n}, \rho u_{t}, K\right)^{t}$. On note $A$ la matrice différentielle associée au système (2) telle que $W_{, t}+\sum_{i=1,2} A_{i} W_{, i}=0$, et on définit $A_{n}$ par $A_{n}=\sum_{i=1,2}\left(M A_{i} M^{-1}\right) n_{i}$

$$
(P R)\left\{\begin{array}{cc}
\partial_{t} W_{n}+A_{n} \partial_{n} W_{n}=0 & \left(\mathcal{S}_{n}\right) \\
W_{n}(x, t=0)=W_{g} & \text { si } x . n<0 \\
W_{n}(x, t=0)=W_{d} & \text { si } x . n>0
\end{array}\right.
$$

Le système différentiel $\left(\mathcal{S}_{n}\right)$ étant hyperbolique (non strictement), on en déduit la solution du problème de Riemann associé (PR) [9]. Les champs caractéristiques sont soit linéairement dégénérés, soit vraiment non linéaires à la condition suffisante que $P(\rho Y)$ soit une fonction convexe du volume spécifique.

La vitesse du son dans l'écoulement bifluide turbulent est notée $c^{\prime}=\sqrt{Y c^{2}+\frac{10}{9} \frac{K}{\rho}}$ et $c^{2}=P^{\prime}(\rho Y)$

$\left(\mathcal{S}_{n}\right)$ admet pour valeurs propres $\lambda_{1}=u_{n}-c^{\prime}, \lambda_{2,3,4}=u_{n}, \lambda_{5}=u_{n}+c^{\prime}$

La solution est constituée d'au plus quatre états constants séparés par des ondes de choc ou détente et une discontinuité de contact correspondant à l'onde triple [9]. Au passage de la discontinuité de contact séparant les états 1 et 2 , les sauts suivants sont nuls, (avec la convention $[\varphi]_{a}^{b}=\varphi(b)-\varphi(a)$ ): $\left[u_{n}\right]_{1}^{2}=0$ et $\left[\frac{2 K}{3}+P\right]_{1}^{2}=0$ La construction des ondes de détente repose sur le calcul des invariants de Riemann. 
On donne l'expression des ondes de détente entre l'état gauche $g$ et l'état $1 \mathcal{D}_{g 1}$ et entre l'état droit $d$ et l'état $2 \mathcal{D}_{d 2}[14]$, avec $c_{k}^{\prime}(x)=\sqrt{Y_{k} c^{2}\left(Y_{k} x\right)+\frac{10}{9} \frac{K_{k}}{\rho_{k}^{5 / 3}} \cdot x^{\frac{2}{3}}}:$

$$
\begin{aligned}
& \mathcal{D}_{g 1}\left(\mathcal{U}_{g}\right)=\left\{\left(\rho, Y, u_{\tau}, K, u_{n}\right), \rho>0, Y=Y_{g}, u_{\tau}=u_{\tau g}, K=\frac{K_{g} \rho^{5 / 3}}{\rho_{g}^{5 / 3}}, u_{n}=u_{n g}+\int_{\rho}^{\rho_{g}} \frac{c_{g}{ }^{\prime}(x)}{x} d x\right\} \\
& \mathcal{D}_{d 2}\left(\mathcal{U}_{d}\right)=\left\{\left(\rho, Y, u_{\tau}, K, u_{n}\right), \rho>0, Y=Y_{d}, u_{\tau}=u_{\tau d}, K=\frac{K_{d} \rho^{5 / 3}}{\rho_{d}^{5 / 3}}, u_{n}=u_{n d}-\int_{\rho}^{\rho_{d}} \frac{c_{d}{ }^{\prime}(x)}{x} d x\right\}
\end{aligned}
$$

Quant aux ondes de choc, elles sont obtenues en écrivant les relations de saut de Rankine-Hugoniot au franchissement d'une discontinuité. Le produit non conservatif $K \partial_{x} u_{n}$ est approché en intégrant le long du chemin des lignes droites [5].

$$
\int_{\mathbf{R}} K \partial_{x} u_{n} d x=\frac{K_{g}+K_{d}}{2}\left[u_{n}\right]
$$

Les ondes de choc sont données avec les mêmes notations $\mathcal{C}_{g 1}$ et $\mathcal{C}_{d 2}$ :

$$
\begin{aligned}
& \mathcal{C}_{g 1}\left(\mathcal{U}_{g}\right)=\left\{\left(\rho, Y, u_{\tau}, K, u_{n}\right), \rho>0, Y=Y_{g}, u_{\tau}=u_{\tau g}, K=\frac{4 \rho-\rho_{g}}{4 \rho_{g}-\rho} K_{g},\right. \\
& \left.u_{n}=u_{n g}-\sqrt{\frac{\left.\left(\rho-\rho_{g}\right)\left[\frac{2}{3}\left(K-K_{g}\right)+P-P_{g}\right]\right)}{\rho \rho_{g}}}\right\} \\
& \mathcal{C}_{d 2}\left(\mathcal{U}_{d}\right)=\left\{\left(\rho, Y, u_{\tau}, K, u_{n}\right), \rho>0, Y=Y_{d}, u_{\tau}=u_{\tau d}, K=\frac{4 \rho-\rho_{d}}{4 \rho_{d}-\rho} K_{d}\right. \\
& \left.u_{n}=u_{n d}+\sqrt{\frac{\left(\rho-\rho_{d}\right)\left[\frac{2}{3}\left(K-K_{d}\right)+\left(P-P_{d}\right)\right]}{\rho \rho_{d}}}\right\}
\end{aligned}
$$

La partie admissible des ondes de choc a été sélectionnée en écrivant la croissance de l'entropie aux chocs. On a donc dû trouver toutes les entropies mathématiques du système, puis on a déterminé parmi celles-ci l'entropie physique du système $\mathcal{E}$ de flux $f_{\mathcal{E}} \cdot \mathcal{E}$ correspond à l'énergie totale du système:

$$
\mathcal{E}=\frac{\rho U^{2}}{2}+K+\rho \int \frac{P(\rho Y)}{\rho^{2}} d \rho \quad, \quad f_{\mathcal{E}}=\frac{\rho U^{2}}{2} U+\frac{5}{3} K U+\rho U \int \frac{P(\rho Y)}{\rho^{2}} d \rho+P U
$$

On démontre que la croissance de l'entropie aux chocs est équivalente à la compressibilité aux chocs [6].

$$
\sigma[\mathcal{E}] \geq\left[f_{\mathcal{E}}\right] \Leftrightarrow[\rho]>0
$$

La démonstration de l'existence de la solution du problème de Riemann repose sur la construction paramètrée de la solution, du même type que celle de la méthode de Smoller [13](p.346-355). On évite cependant de paramétrer le passage des états gauche et droit aux états intermédiaires par des fonctions exponentielles, trop coûteuses dans les implémentations informatiques. La résolution du problème de Riemann de façon exacte se ramène alors à un problème de recherche du zéro d'une fonction non linéaire de $\mathbf{R}^{2}$, qui grâce à ses propriétés de monotonie par rapport à ses deux arguments, permet une résolution numérique par une méthode de type Newton [7][6].

On cherche à résoudre ce système sur un maillage $\Omega_{i=1, \ldots, N}\left(\Omega=\cup_{i} \Omega_{i}, \Omega_{i} \cap \Omega_{j}=\emptyset\right.$ si $i \neq j$, $i \in\{1, \ldots, N\})$. Soit $W_{i}^{n}$ la solution approchée sur la maille $\Omega_{i}, W_{i}^{n}=\frac{1}{\left|\Omega_{i}\right|} \int_{\Omega_{i}} W(x, n \Delta t) d x$ où $\Delta t$ est le pas de temps soumis à une condition CFL. La construction de la solution exacte $W^{R}$ du problème de 
Riemann, est nécessaire au calcul des flux du schéma de Godunov [10]. On note $W_{g d}^{*}=\left(C_{g d}^{*}, K_{g d}^{*}\right)=$ $W^{R}\left(W_{g}, W_{d}, \frac{x}{t}=0\right), l_{i j}=\left|\partial \Omega_{i} \cap \partial \Omega_{j}\right|$ et $V(i)$ est l'ensemble des cellules voisines de la maille $\Omega_{i} . n_{i j}$ est la normale à l'interface des mailles $\Omega_{i}, \Omega_{j}$, orientée de la maille $\Omega_{i}$ vers $\Omega_{j}$.

$$
\begin{gathered}
\overline{K_{i}}=\left(\sum_{j \in V(i)} K_{i j}^{*}\right) /\left(\sum_{j \in V(i)} 1\right) \\
\left\{\begin{array}{l}
C_{i}^{n+1}=C_{i}^{n}-\frac{\Delta t}{\left|\Omega_{i}\right|} \sum_{j \in V(i)} F\left(W_{i j}^{*}\right) \cdot n_{i j} l_{i j} \\
K_{i}^{n+1}=K_{i}^{n}-\frac{\Delta t}{\left|\Omega_{i}\right|} \sum_{j \in V(i)}\left(K_{i j}^{*} u_{n_{i j}}^{*}+\frac{2}{3} \overline{K_{i}} u_{n_{i j}}^{*}\right) l_{i j}
\end{array}\right.
\end{gathered}
$$

Cette méthode numérique s'applique sur tout type de maillage, structuré ou non [7].

\section{Le schéma Vfroe-ncv}

Une confrontation du schéma de Godunov à des solveurs approchés permet de rendre compte de l'intérêt d'utiliser un solveur exact même plus coûteux. On s'est intéressé tout d'abord au solveur linéarisé du schéma Vfroe-ncv. Le schéma Vfroe a été introduit en 1996 dans [8]. Il est basé sur une résolution locale du problème de Riemann linéarisé. Une extension de ce schéma aux variables non conservatives a été faite dans [3]. Ici, pour préserver les invariants de Riemann aux franchissements des discontinuités de contact, nous prendrons la variable $Z=\left(Y, u_{n}, u_{t}, K, P\right)^{t}, \tau=\frac{1}{\rho}$. On résout le problème de Riemann linéarisé $(P R L)$ associé à la matrice constante $A_{n}(\hat{Z})$ avec $\hat{Z}=\frac{Z_{g}+Z_{d}}{2}$, moyenne des états $g$ et $d$.

$$
(P R L) \begin{cases}\partial_{t} Z+A_{n}(\hat{Z}) \partial_{n} Z=0 & \text { si } x . n<0 \\ Z=Z_{g} & \text { si } x . n>0 \\ Z=Z_{d} & \end{cases}
$$

Avec $\hat{c}^{\prime 2}=\left(\gamma \hat{P}+\frac{10}{9} \hat{K}\right) \hat{\tau}$, les valeurs propres de $A_{n}(\hat{Z})$ sont $\lambda_{1}(\hat{Z})=\hat{u}_{n}-\hat{c}^{\prime}, \lambda_{2,3,4}=\hat{u}_{n}, \lambda_{5}=\hat{u}_{n}+\hat{c}^{\prime}$

$$
Z_{d}=Z_{g}+\sum_{i=1,5} \alpha_{i}(\hat{Z}) r_{i}(\hat{Z})
$$

On note $Z_{g d}^{*}\left(Z_{g}, Z_{d}\right)$ la solution à l'interface $\frac{x}{t}=0 \mathrm{du}(P R L)$, avec $Z_{1}$ et $Z_{2}$ les deux états intermédiaires.

$$
\begin{gathered}
\left\{\begin{array}{cc}
Z_{g d}^{*}=Z_{g} \quad \text { si } \hat{u}_{n}-\hat{c}^{\prime}>0 \\
Z_{g d}^{*}=Z_{1} \quad \text { si } 0<\hat{u}_{n}<\hat{c}^{\prime} \\
Z_{g d}^{*}=Z_{2} \quad \text { si }-\hat{c}^{\prime}<\hat{u}_{n}<0 \\
Z_{g d}^{*}=Z_{d} \quad \text { si } \hat{u}_{n}+\hat{c}^{\prime}<0
\end{array}\right. \\
\left\{\begin{array}{l}
Z_{1}=Z_{g}+\frac{1}{2 \hat{c}^{\prime}}\left\{\left[u_{n}\right]_{g}^{d}-\frac{\hat{\tau}}{\hat{c}^{\prime}}\left[\frac{2}{3} K+P\right]_{g}^{d}\right\} r_{1}(\hat{Z}) \\
Z_{2}=Z_{d}-\frac{1}{2 \hat{c}^{\prime}}\left\{\left[u_{n}\right]_{g}^{d}+\frac{\hat{\tau}}{\hat{c}^{\prime}}\left[\frac{2}{3} K+P\right]_{g}^{d}\right\} r_{5}(\hat{Z})
\end{array}\right.
\end{gathered}
$$

L'extension du schéma Vfroe au système non conservatif est donnée par:

$$
\left\{\begin{array}{l}
C_{i}^{n+1}=C_{i}^{n}-\frac{\Delta t}{\left|\Omega_{i}\right|} \sum_{j \in V(i)} F\left(Z_{i j}^{*}\right) n_{i j} l_{i j} \\
K_{i}^{n+1}=K_{i}^{n}-\frac{\Delta t}{\left|\Omega_{i}\right|} \sum_{j \in V(i)}\left(K_{i j}^{*} u_{n_{i j}}^{*}+\frac{2}{3} \bar{K}_{i} u_{n_{i j}}^{*}\right) l_{i j}
\end{array}\right.
$$

Le solveur linéarisé peut générer des états intermédiaires qui ne respectent pas la positivité des variables physiques $\rho, P$ ou $K$. Par exemple, à l'état miroir d'une double raréfaction symétrique (correspondant à une condition limite de paroi en détente) pour les conditions initiales suivantes: $\left(Y, u_{n}, u_{t}, K, P\right)$ 
pour $x<0$ et $\left(Y,-u_{n}, u_{t}, K, P\right)$ pour $x>0$ avec $u_{n}<0, \vec{n}$ étant la normale sortante, on obtient:

$$
K_{1}=K\left(1+\frac{5}{3} \frac{u_{n}}{\hat{c}^{\prime}}\right), \quad P_{1}=P\left(1+\gamma \frac{u_{n}}{\hat{c}^{\prime}}\right) \text { donc } K_{1}<0 \text { pour } \frac{u_{n}}{\hat{c}^{\prime}}<-\frac{5}{3} \text { et } P_{1}<0 \text { pour } \frac{u_{n}}{\hat{c}^{\prime}}<-\frac{1}{\gamma}
$$

De plus, on ne peut pas garantir la positivité de maille des valeurs $\rho, Y, P, K$.

\section{Le schéma de Rusanov}

On considère l'écriture (19) d'un système non conservatif et on définit alors $\tilde{A}_{n}$ par (20).

$$
\begin{gathered}
\partial_{t} W+\sum_{i=1,2}\left(H_{i}(W)\right)_{, i}+\sum_{i=1,2} B_{i} W_{, i}=0 \\
\tilde{A}_{n}=\sum_{i=1,2} \frac{\partial H_{i}(W)}{\partial W} \cdot n_{i}+\sum_{i=1,2} n_{i} B_{i}
\end{gathered}
$$

On introduit $\tilde{S}_{i, j}=\max _{i, j}\left(\max _{k}\left|\lambda_{k}\left(W_{i}\right)\right|,\left|\lambda_{k}\left(W_{j}\right)\right|\right)$ avec $\lambda_{k}$ les valeurs propres de $\tilde{A}_{n}$,

et $W_{i j}=\frac{W_{i}+W_{j}}{2}$. Le schéma de Rusanov, appliqué à notre système $(\mathcal{S})$ s'écrit, avec $\mathcal{F}^{R}$ défini par $(22)$ :

$$
\left\{\begin{array}{c}
C_{i}^{n+1}=C_{i}^{n}-\frac{\Delta t}{\left|\Omega_{i}\right|} \sum_{j \in V(i)} \mathcal{F}^{R}\left(W_{i}^{n}, W_{j}^{n}, n_{i j}\right) l_{i j} \\
K_{i}^{n+1}=K_{i}^{n}-\frac{\Delta t}{\left|\Omega_{i}\right|} \sum_{j \in V(i)}\left(\left(K u_{n}\right)_{i j}-\frac{1}{2} \tilde{S}_{i j}\left(K_{j}-K_{i}\right)+\frac{2}{3} K_{i} u_{i j} n_{i j}\right)^{n} l_{i j} \\
\mathcal{F}^{R}\left(W_{i}, W_{j}, n_{i j}\right)=\frac{1}{2}\left(F\left(W_{i}\right) n_{i j}+F\left(W_{j}\right) n_{i j}-\tilde{S}_{i, j}\left(C_{j}-C_{i}\right)\right)
\end{array}\right.
$$

Le schéma de Rusanov a été choisi pour notre système parce qu'il respecte la positivité de maille [14] de $\rho, \rho Y$ et $K$ et parce qu'il assure également à la fraction massique de rester comprise dans le bon ensemble $0<Y<1$, sous condition C.F.L. : $\Delta t \sum_{j \in V(i)}\left(\tilde{S}_{i j}+\frac{2}{3} u_{j} n_{i j}\right) l_{i j}<2\left|\Omega_{i}\right|$

\section{Résultats numériques}

Grâce au schéma linéarisé Vfroe, le temps de calcul se trouve divisé par 10, tout en donnant une bonne précision des résultats. Si l'on compare la norme $L^{2}$ d'une solution 2D avec celle calculée par la méthode de Godunov on constate un pourcentage d'erreur inférieur à 1.5. Avec le schéma de Rusanov, on divise encore le temps de calcul d'un facteur 3 . De plus, le principe du maximum pour la concentration est respecté. On traite le cas d'une tuyère à rétrécissements (cf. FIG. 1) pour des conditions initiales: $(\rho, Y, u, v, K, P)=(1.1,1,600,0,1000,100000)$ sur maillage régulier de 600 quadrangles. Ce cas test révèle les avantages et inconvénients des différentes méthodes. Avec le schéma de Rusanov aucun problème de positivité n'apparait, mais l'évaluation de $K$ se révèle assez approximative. Quant au schéma Vfroe-ncv, il donne de bons résultats, mais pour le traitement des fortes détentes, notamment lors de simulation d'arrière corps où l'écoulement se raréfie, il est préférable d'utiliser le schéma de Godunov pour garantir la positivité de la densité et de la turbulence. On donne ici une comparaison de la turbulence calculée par la méthode de Godunov avec celle obtenue par le schéma Vfroe-ncv. On donne en a) la turbulence calculée par le schéma de Godunov et en b) le pourcentage d'écart, calculé sur la turbulence, du schéma Vfroe-ncv par rapport au schéma de Godunov.

\section{Conclusion}

Nous préconisons, en général, une utilisation du solveur Vfroe corrélée à la résolution par solveur exact pour le traitement des frontières du domaine. Pour certains cas physiques plus "pathologiques" (fortes détentes), nous préférerons le schéma de Godunov, plus robuste. Ce travail se poursuit par la prise 
en compte des termes visqueux (laminaires et turbulents) tout en gardant un traitement convectif couplé (pression-turbulence). Un travail similaire, sur un modèle de turbulence au second ordre, a montré la supériorité d'une telle approche couplée, par rapport à une approche numérique basée sur un découplage des équations, de fortes instabilités au niveau des conditions limites de paroi apparaissant avec le modèle découplé [4].
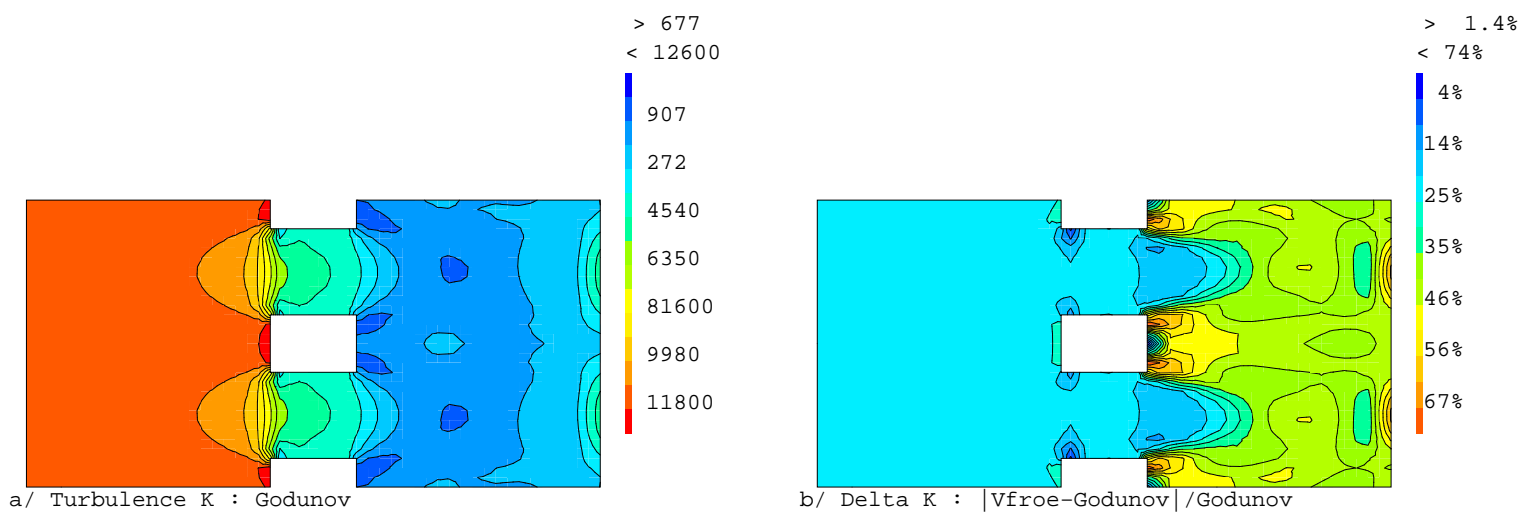

FIG. 1 - a) K par le schéma de Godunov b) Écart sur K entre Vfroe-ncv et Godunov

Fig.a) K by Godunov scheme Fig. b) Difference between K by Vfroe-ncv and K by Godunov scheme

\section{Références bibliographiques}

[1] Berthon C., 1999. Contribution à l'analyse numérique des équations de Navier-Stokes compressibles à deux entropies spécifiques. Application à la turbulence compressible, Thèse de Doctorat, Université Paris VI.

[2] Berthon C., Coquel F., Convection diffusion system with first and second order in nonconservation form, en préparation.

[3] Buffard T., Gallouët T., Hérard J.M. A sequel to a rough Godunov scheme: application to real gases, à paraître dans Computers and Fluids.

[4] Brun G., Hérard J.M., Jeandel D., Uhlmann M. An approximate Roe-type Riemann solver for a class of realizable second order closures, à paraître dans International Journal of Computational Fluid Dynamics.

[5] Dal Maso G., Le Floch P., Murat F., 1995. Definition and weak stability of nonconservative products, J. Math. Pures et Appliquées, Vol. $74 n^{\circ} 6$ pp.483-54.

[6] Declercq E., Forestier A., Hérard J.M.H. et al., 1999. Part 1: An exact Riemann solver for a multicomponent turbulent flow, Part 2: Comparison of numerical solvers for a multicomponent turbulent flow, soumis en version corrigée à International Journal of Computational Fluid Dynamics.

[7] Forestier A. , Hérard J.M., Louis X. , 1997. Solveur de type Godunov pour simuler les écoulements turbulents compressibles, C.R.A.S. Paris, t. 324, Série I, pp.919-926.

[8] Gallouet T. , Masella J.M., 1996. Un schéma de Godunov approché, C.R.A.S. Paris, t.323, Série I, pp.77-84.

[9] Godlewski E., Raviart P.A., 1996. Numerical Approximation of Hyperbolic Systems of Conservation Laws, Applied Mathematical Sciences 118, ED. Springer.

[10] Godunov S. K., 1959. A difference method for numerical calculation of discontinous equations of hydrodynamics, Math. Sb., 47, pp. 217-300.

[11] Mohammadi B., Pironneau 0., 1994. Analysis of the K-Epsilon Turbulence Model, Masson.

[12] Rusanov V., 1961. Calculation of interaction of non-steady schok waves with obstacles, Journal of Computational Mathematics and Physics USSR, Vol 1, pp.267-279.

[13] Smoller J., 1983. Shock waves and reaction-diffusion equations, Springer-Verlag.

[14] Xeuxet-Declercq E., 1999. Comparaison de solveurs numériques pour le traitement de la turbulence bi-fluide, Thèse de Doctorat, Université d'Evry, 23/6/1999. 